\title{
Hall-Effect Sensor Technique for No Induced Voltage in AC Magnetic Field Measurements Without Current Spinning
}

\author{
A. V. Lalwani ${ }^{1}$, A. S. Yalamarthy ${ }^{2}$, H. S. Alpert ${ }^{3}$, M. A. Holliday ${ }^{4}$, S. R. Eisner ${ }^{1}$, C. A. Chapin ${ }^{2,3}$, \\ D. G. Senesky, Member IEEE $E^{1,3}$ \\ ${ }^{1}$ Department of Electrical Engineering, Stanford University, Stanford, CA 94305 USA \\ ${ }^{2}$ Department of Mechanical Engineering, Stanford University, Stanford, CA 94305 USA \\ ${ }^{3}$ Department of Aeronautics and Astronautics, Stanford University, Stanford, CA 94305 USA \\ ${ }^{4}$ Department of Material Science and Engineering, Stanford University, Stanford, CA 94305 USA
}

\begin{abstract}
Accurately sensing AC magnetic field signatures poses a series of challenges to commonly used Hall-effect sensors. In particular, induced voltage and lack of high-frequency spinning methods are bottlenecks in the measurement of $\mathrm{AC}$ magnetic fields. We describe a magnetic field measurement technique that can be implemented in two ways: 1) the current driving the Hall-effect sensor is oscillating at the same frequency as the magnetic field, and the signal is measured at the second harmonic of the magnetic field frequency, and 2) the frequency of the driving current is preset, and the measured frequency is the magnetic field frequency plus the frequency of the current. This method has potential advantages over traditional means of measuring AC magnetic fields used in power systems (e.g., motors, inverters), as it can reduce the components needed (subsequently reducing the overall cost and size) and is not frequency bandwidth limited by current spinning. The sensing technique produces no induced voltage and results in a low offset, thus preserving accuracy and precision in measurements. Experimentally, we have shown offset voltage values between 8 and $27 \mu$ T at frequencies ranging from $100 \mathrm{~Hz}$ to $1 \mathrm{kHz}$, validating the potential of this technique in both cases.
\end{abstract}

\section{INTRODUCTION}

Hall-effect sensors are used to measure the magnetic field in a variety of applications, including electricity generation at power stations, electric motors, and power electronics. The wide diversity of applications of Hall-effect sensors has driven significant research on device geometry, noise reduction, current-spinning methodology, and driving circuitry, with the primary goals of reducing noise, improving accuracy, and decreasing the cost of Hall-effect sensors [1-3].

In measurements taken with Hall-effect sensors, the minimum detectable magnetic flux density depends on the noise as well as the offset voltage, which is the voltage that appears at the plate output contacts when the magnetic field stimulus is 0. A typical Hall-effect plate will always show a non-zero offset voltage because of resistance gradients, geometrical asymmetries arising from manufacturing tolerances, thermal gradients, or piezoresistive effects [4]. One of the most significant advances in Hall-effect sensor accuracy is the current-spinning method, which is used to significantly reduce offset voltage [4]. In this method, the direction of the current flowing across the Hall-effect sensor is switched from one pair of contacts to the other, effectively cancelling most of the offset. A small offset, called the residual offset, is still present after current spinning and is typically between hundreds of $\mu \mathrm{T}$ to tens of nT [5-7].

However, many applications require Hall-effect sensors to operate in AC magnetic fields, which poses challenges for current-spinning. In a rapidly oscillating magnetic field, the current direction must switch faster than the magnetic field to enable offset cancellation. The current spinning of Hall-effect devices is generally limited to $250 \mathrm{kHz}$ in the fastest-spinning circuits in the literature $[8,9]$. The few cases reported that have higher bandwidth rely on current transformers or coils, thus adding to the overall cost and size of the system [10-12]. New techniques in magnetic sensing, such as tunneling magnetoresistive (TMR) can achieve higher frequency bandwidths but are limited in detection of the overall range of the magnitude of the magnetic field [13]. The use of Hall-effect sensors in high frequency applications is limited by their intrinsic capacitance (the practical limit) and current spinning (the methodological limit), where the practical limit is higher than the 
methodological limit [14]. The current bottleneck for Halleffect sensors to operate at high frequencies is current spinning. As such, there is a demonstrated need for small, low-cost, large bandwidth Hall-effect sensors that are capable of measuring frequencies in the $\mathrm{MHz}$ range, for use in power electronics, electric motors, control of inverters and magnetic resonance imaging (MRI) applications $[15,16]$. It should be noted that there are applications in which the magnetic field frequency is known (e.g., transformers, MRI machines) and also cases in which the magnetic field frequency is unknown (e.g., electric engines, turbines). The proposed techniques in this paper address both of these cases.

Moreover, a difficulty with measuring AC magnetic fields is the induced voltage. The output of a Hall-effect sensor is the voltage across two terminals ( $V_{\text {OUT }}$ ); therefore, in the presence of a changing magnetic field, the Hall-effect sensor has an induced voltage due to Faraday's Law. It is useful to note that the induced voltage is at the same frequency as the magnetic field being measured, while offset voltage is in DC.

The specific technique described in this paper is general for all Hall-effect sensors; however, this paper is based on experimentation with an indium aluminum nitride on gallium nitride (InAlN/GaN) Hall-effect sensor [2]. For DC magnetic fields, the voltage output of the Hall-effect sensor is:

$$
V_{\text {OUT }}=\frac{I(B+\alpha)}{q n_{s}}
$$

where $V_{\text {OUT }}$ is the measured voltage, $I$ is the current driving the Hall-effect sensor, $B$ is the external magnetic field, $\alpha$ is the offset, $q$ is electronic charge constant, and $n_{s}$ is the sheet density of electrons in the two-dimensional electron gas (2DEG) at the InAlN/GaN interface. This equation is the Hall-effect voltage equation, modified to include the offset.

In addition, for magnetic fields oscillating at frequency $\omega$, there is an added term for the induced magnetic field, leading to an induced voltage due to Faraday's Law:

$$
\begin{gathered}
B=B_{o} \sin (\omega t) \\
V_{\text {OUT }}=\frac{I\left(B_{o} \sin (\omega t)+\alpha\right)}{q n_{s}}+\beta \frac{d B}{d t}
\end{gathered}
$$

where $B_{o}$ is the magnetic field magnitude and $\varphi$ is the phase of the magnetic field. The induced voltage term is proportional to the inductance of the sample and does not exist in nonoscillating magnetic fields. We denoted $\beta$ as the constant of proportionality.

To eliminate the induced voltage, a commonly used method is the dual Hall-effect plate technique [17]. This method relies on two Hall-effect sensors in operation. The first Hall-effect sensor is driven at a static current and current-spinning is applied. The second Hall-effect sensor captures the induced voltage, $\beta \frac{d B}{d t}$, by operating at zero current. Then, the induced voltage is subtracted from the signal of the first Hall-effect sensor.
Other techniques to measure AC magnetic fields, such as current transformers, are often cost-prohibitive and sizeprohibitive for wide-scale implementation. There is a clear need for small, cost-effective AC magnetic field sensors [17-20]. Here, we describe a technique that enables a single Hall-effect sensor to measure high-frequency magnetic fields with low offset voltage and no induced voltage.

\section{CASE 1: KNOWN MAGNETIC FIELD FREQUENCY}

In the following technique, an AC current is applied to the Hall-effect sensor at the same frequency as the magnetic field being measured. The generated signal is then measured at double the frequency (2- $\omega)$ of the input current, resulting in a signal of just the magnetic field magnitude with no induced AC voltage, offset voltage, or phase mismatch. This technique is an adaptation of a technique used for non-destructive evaluation of electrical properties of semiconductors and is similar to the technique used in synchronous modulation of signals, but it has not been applied to measure AC magnetic fields [21]. A schematic representation of the method is shown in graphical abstract.

Using the oscillating signals, the $\mathrm{AC}$ magnetic field $(B)$ and driving AC current $(I)$ can be written as

$$
\begin{gathered}
I=I_{o} \sin (\omega t) \\
B=B_{o} \sin (\omega t+\varphi)
\end{gathered}
$$

where the frequency of the two signals is the same and the phase mismatch is denoted by $\varphi$. Substituting these terms into (3), the resulting output voltage is:

$$
\begin{gathered}
V_{\text {OUT }}=\frac{I_{o} \sin (\omega t)\left(B_{o} \sin (\omega t+\varphi)+\alpha\right)}{q n_{s}}+\beta \omega B_{o} \cos (\omega t+\varphi) \\
+\frac{I_{o} \sin (\omega t)\left(B_{o} \sin (\omega t) \cos (\varphi)+B_{o} \sin (\varphi) \cos (\omega t)+\alpha\right)}{q n_{s}}(7) \\
+\beta \omega B_{o} \cos (\omega t+\varphi) \\
=\frac{I_{o} B_{o} \sin ^{2}(\omega t) \cos (\varphi)+I_{o} B_{o} \sin (\omega t) \sin (\varphi) \cos (\omega t)+\alpha I_{o} \sin (\omega t)}{q n_{s}} \\
+\beta \omega B_{o} \cos (\omega t+\varphi)
\end{gathered}
$$

Using the double angle formula identity of $\sin ^{2}(x)$ : 


$$
\begin{gathered}
=-\frac{I_{o} B_{o} \cos (2 \omega t) \cos (\varphi)}{2 q n_{s}}+\frac{I_{o} B_{o} \sin (2 \omega t) \sin (\varphi)}{2 q n_{s}}+\frac{\alpha I_{o} \sin (\omega t)}{q n_{s}} \\
+\beta \omega B_{o} \cos (\omega t+\varphi)+\frac{I_{o} B_{o} \cos (\varphi)}{2 q n_{s}}
\end{gathered}
$$

Using the sum and difference formulae for sine and cosine:

$$
\begin{gathered}
V_{\text {out }}=-\frac{I_{o} B_{o} \cos (2 \omega t+\varphi)}{2 q n_{s}}+\frac{\alpha I_{o} \sin (\omega t)}{q n_{s}}+\beta \omega B_{o} \cos (\omega t+\varphi) \\
+\frac{I_{o} B_{o} \cos (\varphi)}{2 q n_{s}}
\end{gathered}
$$

Equation (10) shows that the portion of the signal proportional to the offset $\alpha$ as well as the induced voltage term $\beta \omega B_{o} \cos (\omega t+\varphi)$ are both at frequency $\omega$. Meanwhile, the signal of interest, $I_{o} B_{o}$, is at $2-\omega$. The phase mismatch between the current driving frequency and magnetic frequency is at DC, as shown in the last term of (10). At 2- $\omega$, the phase is captured inside the cosine term, thus not having an effect on the overall magnitude of the signal. Using bandpass filters or a lock-inamplifier, the signal produced at 2- $\omega$ can be isolated from the induced voltage and the offset at $\omega$. The sensitivity of the Halleffect sensor at 2- $\omega$ is defined as $\frac{I_{o}}{2 q n_{s}}$. The proposed technique does not require current spinning or a secondary Hall-effect plate to counter the induced magnetic field and the DC offset, and thereby has the potential to reduce the cost and size of the overall device. Measurement at $0 \mathrm{~Hz}$ is problematic, as the phase mismatch, which is an artifact of the setup that is unknown and difficult to get rid of, will affect the overall signal - thus making DC measurements unreliable.

\section{CASE 1: SIMULATION}

We simulated the 2- $\omega$ technique with an $\mathrm{AC}$ magnetic field at a frequency of $500 \mathrm{~Hz}$, an input current also at $500 \mathrm{~Hz}$, and a phase mismatch varying from 0 to 90 degrees. The measured signal was deconvoluted using the fast Fourier transform function, and the 2- $\omega(1000 \mathrm{~Hz})$ component was isolated from the $\omega(500 \mathrm{~Hz})$ component. The results are displayed in Fig. 1.

Fig. 1 shows the three components of the output voltage due to (10) measured at $500 \mathrm{~Hz}$ (Fig. 1a) and $1000 \mathrm{~Hz}$ (Fig. 1b). The green line is the portion of the output voltage (amplitude) due to the external magnetic field $\frac{-I_{O} B_{o} \cos (2 \omega t+\varphi)}{2 q n_{S}}$ as $B_{o}$ is varied, the red line is the portion of the output due to the induced magnetic field $\beta B_{o} \omega \cos (\omega t+\varphi)$, and the blue line is the portion of the output voltage due to the offset $\frac{I_{o} \alpha \sin (\omega t)}{q n_{s}}$. The offset, $\alpha$, was held constant in the simulation at $5.5 \mu \mathrm{V}$ and the magnitude of the induced voltage, $\beta$, was held at $1 \mathrm{~V} \cdot \mathrm{s} / \mathrm{mT}$. Sheet density, $n_{s}$, was $2.1 \times 10^{13} \mathrm{~cm}^{-2}$, as measured experimentally for the InAlN/GaN Hall-effect sensor at room temperature [2].
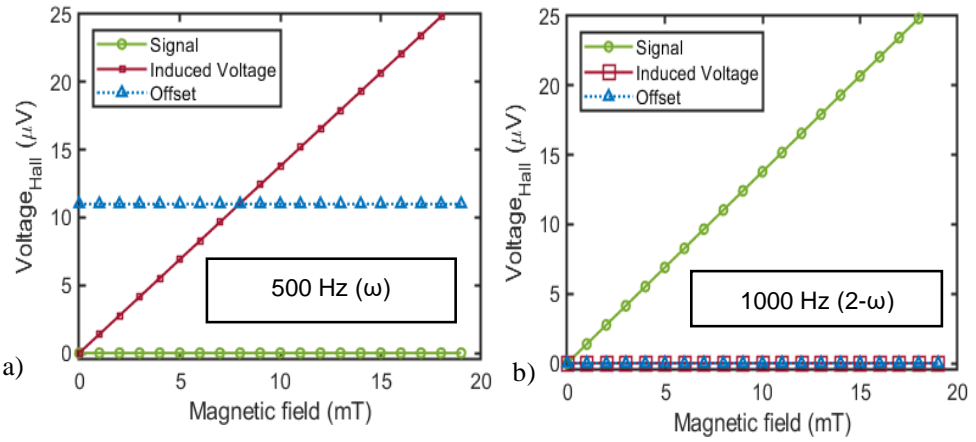

Fig. 1. Simulations of a) output voltage measured at $500 \mathrm{~Hz}(\omega)$, and b) output voltage measured at $1000 \mathrm{~Hz}(2-\omega)$. The output voltages shown here are the signal amplitudes.

It is observed that at $2-\omega$ the output voltage increases linearly with an increase in $B_{o}$ and is not affected by the magnitude of $\alpha$ and $\beta$. At $\omega$, the external magnetic field has a linear relationship with the induced voltage and a constant offset. Varying the phase mismatch did not affect any of the terms at $\omega$ or $2-\omega$.

Being able to isolate the signal from the offset and induced voltage presents a significant advantage over the current stateof-the-art solutions. In practice, the isolation can be achieved using bandpass filters.

\section{CASE 1: EXPERIMENTS AND DISCUSSION}

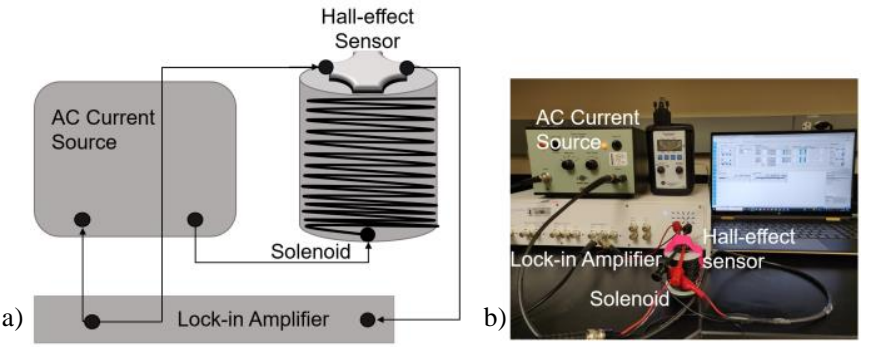

Fig. 2. Experimental setup for testing the $2-\omega$ method. a) line diagram, b) photo of the setup.

To generate the AC magnetic field, a Zurich Instruments lock-in amplifier generated a sine wave, which was amplified by an AC current amplifier (Bruel \& Kjaer). An experimental setup is shown in Fig 2. The output of the amplifier was connected to a solenoid that produced the magnetic field. The solenoid used is a 10-meter, 14 gauge insulated copper wire wound over a plastic cylinder. The output of the lock-in amplifier was connected to an AC current amplifier and an InAlN/GaN Hall-effect sensor [2], to maintain frequency. The InAlN/GaN Hall-effect sensor is a 4-contact sensor with a twodimensional electron gas (2DEG) acting as the conducting layer and was fabricated at Stanford Nanofabrication Facility by Alpert et al. [2]. The Hall-effect sensor was driven by the same lock-in amplifier at $0.5 \mathrm{~V}$ at the same frequency as the magnetic 
field and at various phase mismatches, and the output voltage was measured by the lock-in amplifier at $2-\omega$. The AC magnetic field was also measured for validation using a commercial gaussmeter (AlphaLab Model GM2), which could measure at frequencies up to $800 \mathrm{~Hz}$ with the probe placed on top of the Hall-effect sensor. The experiment was conducted in the presence of Earth's magnetic field and the external magnetic field strength was varied by using the gain of the current amplifier. The frequency of the magnetic field and input current to the Hall-effect sensor, along with the phase mismatch, were varied using the lock-in amplifier. The measured voltage was scaled by the RMS value to report amplitude.

Fig. 3 shows the relationship between the external magnetic field measured with the commercial gaussmeter and the output voltage measured with the lock-in amplifier. The linear relationship obtained demonstrates the validity of the 2$\omega$ technique. The experiment was conducted at various frequencies between 500 and $800 \mathrm{~Hz}$ to further demonstrate that the 2- $\omega$ technique can work across frequencies. Varying the phase of the magnetic field with respect to the current had no effect on the measured output voltages at 2- $\omega$. The average gradient (sensitivity) of the experimental lines in Fig. 3 is 18.49 $\mathrm{mV} / \mathrm{T}$ at R-squared value of 0.999 . Using the values from [2] and above, the theoretical gradient is calculated to be 19.33 $\mathrm{mV} / \mathrm{T}$, which is within $5 \%$ of the experimental value. The offset measured is between 24 and $31 \mu \mathrm{T}$.

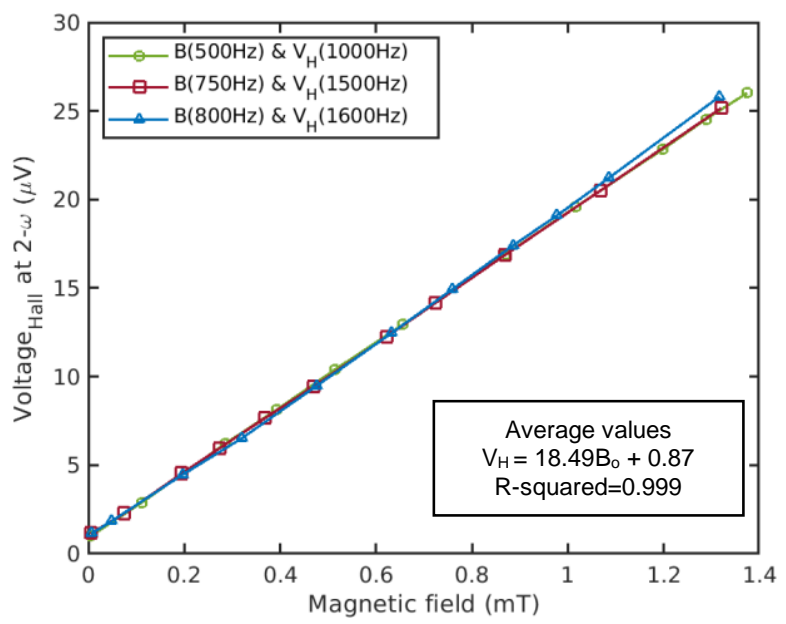

Fig. 3. Output voltage of Hall-effect sensor at frequency of 2- $\omega$ vs. external magnetic field for various driving frequencies.

To experimentally compare the magnitude of the induced voltage between the signals measured at $\omega$ and $2-\omega$, the following experiment was performed. The Hall-effect sensor was disconnected from the current source $\left(I_{o}=0\right)$ and the magnetic field magnitude $\left(B_{o}\right)$ was varied at $800 \mathrm{~Hz}$. The output from the Hall-effect sensor was measured at $800 \mathrm{~Hz}(\omega)$ and $1600 \mathrm{~Hz}(2-\omega)$ and phase mismatch was held constant at $30^{\circ}$. The magnetic field was increased and decreased stepwise with 5 -second intervals.

With zero input current $\left(I_{\mathrm{o}}=0\right)$, only the induced magnetic field component, $\beta B_{o} \cos (\omega t)$, is non-zero in the output voltage signal. Because the induced voltage component oscillates at frequency $\omega$, we would expect to be able to detect a signal at $\omega$ but not at $2-\omega$.

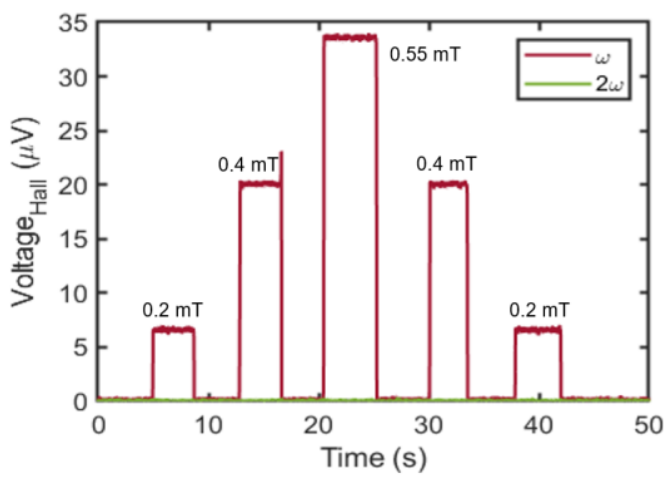

Fig. 4. Output voltage of Hall-effect sensors with no input current $\left(I_{0}=0\right)$, measured at $\omega$ and 2- $\omega$ frequencies.

Fig. 4 shows that the $\omega$ signal (red line) has a strong response to the change in magnetic fields, whereas the signal at $2-\omega$ (green line), has zero response to the change in the magnetic field, as would be expected given the absence of an input current $\left(I_{0}=0\right)$. This result verifies that the signal measured at $2-\omega$ is not significantly affected by voltage due to an induced magnetic field at this frequency, significantly improving the signal-to-noise ratio.

\section{CASE 2: UNKNOWN MAGNETIC FIELD FREQUENCY}

There are applications in which the magnetic field frequency is unknown, or varies over time, such as in electric engines and turbines. In such cases, AC magnetic field measurement can still be achieved using the technique mentioned above, with slight modifications - primarily setting the driving electric current frequency to a constant value and then measuring the magnetic field frequency above the driving current frequency.

Like the equations above, the current driving frequency can be set at a value $\theta$, while the magnetic field frequency is unknown at $\omega$. The phase mismatch is captured by $\varphi$ :

$$
\begin{aligned}
& I=I_{o} \sin (\theta t) \\
& B=B_{o} \sin (\omega t+\varphi)
\end{aligned}
$$

Substituting above equation into (1), the output voltage is:

$$
V_{\text {OUT }}=\frac{I_{o} \sin (\theta t)\left(B_{o} \sin (\omega t+\varphi)+\alpha\right)}{q n_{s}}+\beta \frac{d B}{d t}
$$




$$
\begin{gathered}
=\frac{I_{o} B_{o} \sin (\theta t) \sin (\omega t+\varphi)+\alpha I_{o} \sin (\theta t)}{q n_{s}} \\
+\beta \omega B_{o} \cos (\omega t+\varphi) \\
=\frac{-I_{o} B_{o}}{2 q n_{s}}[\cos (\theta t+\omega t+\varphi)-\cos (\theta t-\omega t-\varphi)]+\frac{\alpha I_{o} \sin (\theta t)}{2 q n_{s}} \\
+\beta \omega B_{o} \cos (\omega t+\varphi)
\end{gathered}
$$

Using the product to sum identity of sine and cosine,

$$
\begin{gathered}
V_{\text {OUT }}=\frac{-I_{o} B_{o} \cos ((\theta+\omega) t+\varphi)}{2 q n_{s}}+\frac{I_{o} B_{o} \cos ((\theta-\omega) t-\varphi)}{2 q n_{s}} \\
+\frac{\alpha I_{o} \sin (\theta t)}{q n_{s}}+\beta \omega B_{o} \cos (\omega t+\varphi)
\end{gathered}
$$

Similar to (10), the above (16) has the offset $\alpha$ at frequency $\theta$, the induced magnetic field $\beta$ at frequency $\omega$, and the signal $I_{o} B_{o}$ at frequency $\omega \pm \theta$. The phase mismatch $\varphi$ does not factor into the magnitude of any of the above equations. This technique enables the measurement of the signal at frequencies different from the noise (offset and induced voltage), thus allowing bandpass filtering techniques to isolate signal from the noise.

\section{CASE 2: SIMULATION}

To illustrate (17), we simulated the $\omega \pm \theta$ technique using the same tools as in the Case 1 simulation. The input magnetic field frequency $\omega$ was held constant at $500 \mathrm{~Hz}$, the current driving frequency $\theta$ was held constant at $300 \mathrm{~Hz}$, and a phase mismatch of $35^{\circ}$ was set. The magnetic field $\left(B_{o}\right)$ was varied between 0 to $6 \mathrm{mT}$. The measured signal was deconvoluted using fast Fourier transform and frequencies of $300 \mathrm{~Hz}, 500 \mathrm{~Hz}$ and $800 \mathrm{~Hz}$ were isolated. The results are displayed in Fig 5.

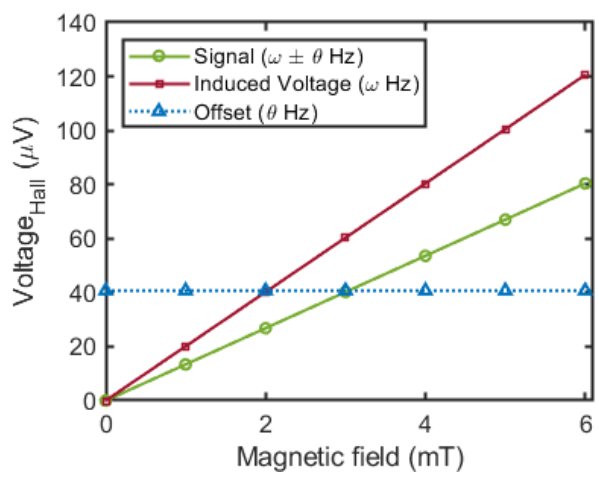

Fig. 5. Simulation of the Hall voltage at three frequencies: $300 \mathrm{~Hz}(\theta), 500 \mathrm{~Hz}$ $(\omega)$ and $800 \mathrm{~Hz}(\omega+\theta)$. The output voltages shown are the signal magnitude at their respective frequencies.

Fig. 5 shows the three components in (16) measured at 300 $\mathrm{Hz}(\theta), 500 \mathrm{~Hz}(\omega)$ and $800 \mathrm{~Hz}(\omega+\theta)$. The green line is the portion of the output voltage (amplitude) due to the external magnetic field $\frac{-I_{o} B_{o} \cos ((\theta+\omega) t+\varphi)}{2 q n_{S}}$ as $B_{o}$ is varied, the red line is the portion of the output due to the induced magnetic field $\beta B_{o} \omega \cos (\omega t+\varphi)$, and the blue line is the portion of the output voltage due to the offset $\frac{I_{o} \alpha \sin (\theta t)}{q n_{s}}$. The offset, $\alpha$, was held constant in the simulation at $21 \mu \mathrm{V}$ and the magnitude of the induced voltage, $\beta$, was held at $1 \mathrm{~V} \cdot \mathrm{s} / \mathrm{mT}$. Sheet density, $n_{s}$, was $2.1 \times 10^{13} \mathrm{~cm}^{-2}$, as measured experimentally for the Halleffect sensor at room temperature [2].

As the external magnetic field is increased, the offset (blue line) measured at $300 \mathrm{~Hz}(\theta)$ has no change, whereas the induced magnetic field (red line) measured at $500 \mathrm{~Hz}(\omega)$ and the signal (green line) measured at $800 \mathrm{~Hz}(\omega+\theta)$ increase linearly with increasing external magnetic field.

\section{CASE 2: EXPERIMENT AND DISCUSSION}

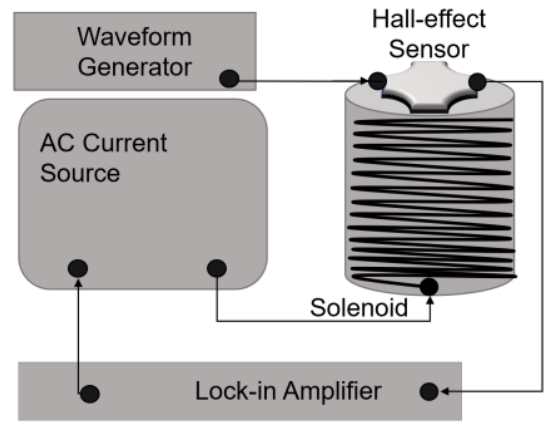

Fig. 6. Experimental setup for the $\omega \pm \theta$ technique.

The experimental setup for the $\omega \pm \theta$ technique is similar to that for the 2- $\omega$ technique. The primary difference is the addition of Agilent 33120A waveform generator, used to provide the current driving signal to the Hall-effect sensor. The lock-in amplifier, AC current source, Hall-effect sensor, commercial gaussmeter and solenoid used are the same as in section IV.

The experiment was conducted in Earth's magnetic field. The current driving frequency was independently controlled by the waveform generator and the magnetic field frequency was controlled by the lock-in amplifier. The strength of the external magnetic field was varied using the AC current source and the Hall-effect voltage was measured using the lock-in amplifier. The magnetic field was varied between $600 \mathrm{~Hz}$ and $1 \mathrm{kHz}$ using the lock-in amplifier, the driving current frequency was varied between $300 \mathrm{~Hz}$ to $1.5 \mathrm{kHz}$ using the waveform generator and at 0.5 peak voltage, and the Hall-effect voltage output was measured between $700 \mathrm{~Hz}$ and $2.1 \mathrm{kHz}$ using the lock-in amplifier. The phase difference between the driving current and magnetic field frequency was measured using Keysight Technologies EDUX1002A oscilloscope. No effect of varying phase was observed in the Hall-effect voltage output. 


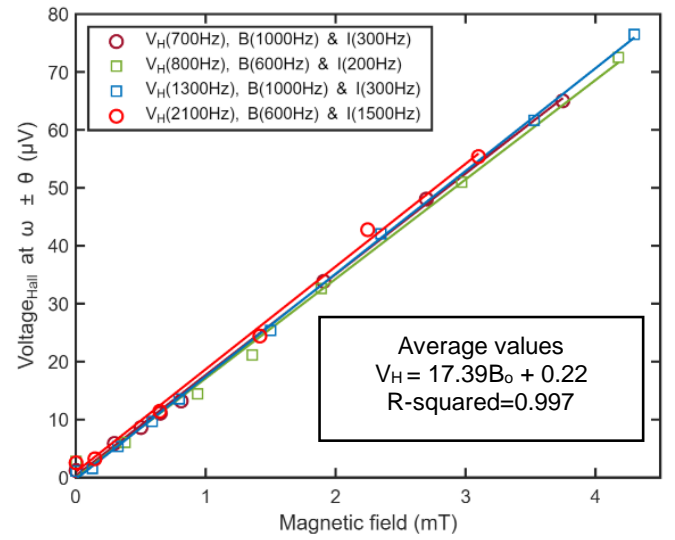

Fig. 7. Hall voltage output measured at $\omega+\theta$ (square) and $\omega-\theta$ (circular) versus external magnetic field at different frequencies.

In Fig. 7 the gradient (sensitivity) is $17.39 \mathrm{mV} / \mathrm{T}$. Using the values from [2] and above, the theoretical gradient is calculated to be $19.33 \mathrm{mV} / \mathrm{T}$, which is within $10 \%$ of the experimental value. The average offset measured in Earth's magnetic field is $8.37 \mu \mathrm{T}$ and R-squared value is 0.997 . Earth's magnetic field ranges from 25-65 $\mu \mathrm{T}$ on the surface. The comparison of sensitivity and offset is captured in Table 1 .

Table 1. Comparing the sensitivity and offset of the 2- $\omega$, $\omega \pm \theta$ and theoretical current spinning.

\begin{tabular}{|c|c|c|}
\hline Technique & $\begin{array}{c}\text { Average sensitivity } \\
(\mathbf{m V} / \mathbf{T})\end{array}$ & $\begin{array}{c}\text { Average offset in } \\
\text { Earth's magnetic field } \\
(\boldsymbol{\mu T})\end{array}$ \\
\hline $2-\omega$ & 18.49 & 27.5 \\
\hline$\omega \pm \theta$ & 17.39 & 8.37 \\
\hline $\begin{array}{c}\text { Theoretical } \\
\text { with current } \\
\text { spinning }\end{array}$ & 19.33 & $25-65$ \\
\hline
\end{tabular}

\section{CONCLUSION}

We have proposed two techniques for operating Hall-effect sensors to measure AC magnetic fields that do not rely on the current-spinning method and result in no induced voltage. The 2- $\omega$ and the $\omega \pm \theta$ techniques have the potential to characterize the frequencies and magnitudes of known and unknown $\mathrm{AC}$ magnetic fields, respectively, with greater accuracy. The techniques also have the potential to operate at much higher frequencies than other widely used methods, as they do not rely on switching circuitry. These techniques may be advantageous in systems with moving parts that generate AC magnetic fields or currents (e.g., turbines, electric motors, transformers). The 2$\omega$ technique can be used if the magnetic field frequency is known, while the $\omega \pm \theta$ technique can be used if the frequency is unknown. Applications for the techniques above range from controlling and monitoring inverters, electric vehicle engines, turbines, and MRI machines.
Future work includes circuit-level implementation of the system and studying the temperature drift of Hall-effect sensors with this technique, as that has proven to be a challenge in the field $[22,23]$. It would also be beneficial to optimize the CMOS integration of the auxiliary components needed for operation of these techniques in order to reduce the overall chip size and costs.

\section{ACKNOWLEDGEMENTS}

Part of this work was performed at the Stanford Nano Shared Facilities (SNSF)/Stanford Nanofabrication Facility (SNF), supported by the National Science Foundation under award ECCS-2026822.

\section{REFERENCES}

[1] A. Bilotti, G. Monreal, and R. Vig, "Monolithic magnetic Hall sensor using dynamic quadrature offset cancellation," IEEE journal of solid-state circuits, vol. 32, no. 6, pp. 829-836, 1997.

[2] H. S. Alpert, K. M. Dowling, C. A. Chapin et al., "Effect of geometry on sensitivity and offset of AlGaN/GaN and InAlN/GaN Hall-effect sensors," IEEE Sensors Journal, vol. 19, no. 10, pp. 3640-3646, 2019.

[3] S.-M. Lim, and J.-S. Park, "A low noise offset cancellation method for improving sensitivity of CMOS Hall sensor," Journal of Electrical Engineering \& Technology, vol. 14, no. 1, pp. 377-383, 2019.

[4] P. Munter, "Electronic circuitry for a smart spinning-current Hall plate with low offset," Sensors and Actuators A: Physical, vol. 27, no. 1-3, pp. 747751,1991

[5] V. Mosser, N. Matringe, and Y. Haddab, "A spinning current circuit for Hall measurements down to the nanotesla range," IEEE Transactions on Instrumentation and Measurement, vol. 66, no. 4, pp. 637-650, 2017.

[6] R. Steiner, C. Maier, A. Haberli et al., "Offset reduction in Hall devices by continuous spinning current method," Sensors and Actuators A: Physical, vol. 66 , no. 1-3, pp. 167-172, 1998 .

[7] R. e. Popovic, Z. Randjelovic, and D. Manic, "Integrated Hall-effect magnetic sensors," Sensors and Actuators A: Physical, vol. 91, no. 1-2, pp 46-50, 2001

[8] J. Jiang, W. J. Kindt, and K. A. Makinwa, "A continuous-time ripple reduction technique for spinning-current Hall sensors," IEEE Journal of solid-state circuits, vol. 49, no. 7, pp. 1525-1534, 2014.

[9] H. Heidari, E. Bonizzoni, U. Gatti et al., "A CMOS current-mode magnetic Hall sensor with integrated front-end," IEEE Transactions on Circuits and Systems I: Regular Papers, vol. 62, no. 5, pp. 1270-1278, 2015.

[10] J. Jiang, and K. A. Makinwa, "Multipath wide-bandwidth CMOS magnetic sensors," IEEE Journal of Solid-State Circuits, vol. 52, no. 1, pp. 198-209, 2016.

[11] J. Wang, D. Si, T. Tian et al., "Design and experimental study of a current transformer with a stacked PCB based on B-dot," Sensors, vol. 17, no. 4, pp. 820, 2017.

[12] M. Crescentini, M. Biondi, M. Bennati et al., "A 2 MS/s 10A Hall current sensor SoC with digital compressive sensing encoder in $0.16 \mu \mathrm{m}$ BCD." pp. 393-396, 42nd European Solid-State Circuits Conference 2016.

[13] S. Hainz, E. de la Torre and J. Güttinger, "Comparison of Magnetic Field Sensor Technologies for the use in Wheel Speed Sensors," 2019 IEEE International Conference on Industrial Technology (ICIT), pp. 727-731, 2019

[14] M. Crescentini, R. Ramilli, G. P. Gibiino et al., "The X-Hall Sensor: Toward Integrated Broadband Current Sensing," IEEE Transactions on Instrumentation and Measurement, vol. 70, pp. 1-12, 2020

[15] C. Fermon, "Introduction on Magnetic Sensing and Spin Electronics," Nanomagnetism: Applications and Perspectives: Applications and Perspectives, pp. 1-18, 2017.

[16] F. Silva, and M. Aragón, "Electromagnetic interferences from electric/hybrid vehicles." XXXth URSI General Assembly and Scientific Symposium, pp. $1-4,2011$.

[17] P. Emerald, "Non-intrusive Hall-Effect current-sensing techniques provide safe, reliable detection and protection for power electronics." pp. 1-2, 1998.

[18] B. Liu, Y. Sun, Y. Ding et al., "Low-power and high-sensitivity system-onchip Hall effect sensor." 2017 IEEE SENSORS Conference Proceedings, pp. pp. 1-3, 2017 
[19] R. Wei, S. Guo, and S. Yang, "Low-Power CMOS Integrated Hall Switch Sensor," Active and Passive Electronic Components, vol. 2017, 2017.

[20] M. Sulowicz, K. Ludwinek, J. Tulicki et al., "Practical adaptation of a lowcost voltage transducer with an open feedback loop for precise measurement of distorted voltages," Sensors, vol. 19, no. 5, pp. 1071, 2019.

[21] H. Weider, "Four-Terminal Nondestructive Electrical and Galvanomagnetic Measurements," Nondestructive evaluation of semiconductor materials and devices, pp. 67-104: Springer, 1979.

[22] R. Prance, T. Clark, and H. Prance, "Ultra low noise induction magnetometer for variable temperature operation," Sensors and Actuators A: Physical, vol. 85, no. 1-3, pp. 361-364, 2000.

[23] I. N. Cholakova, T. B. Takov, R. T. Tsankov et al., "Temperature influence on Hall effect sensors characteristics." 2012 20th Telecommunications

Forum (TELFOR), pp. 967-970, 2012. 